06

\title{
Генерация второй гармоники-суммарной частоты в тонком сферическом слое: III. Свойства решения
}

\author{
(C) А.А. Шамына, В.Н. Капшай, А.И. Толкачёв \\ Гомельский государственный университет им. Ф. Скорины, \\ 246019 Гомель, Республика Беларусь \\ e-mail: anton.shamyna@gmail.com, kapshai@rambler.ru, anton.talkachov@gmail.com
}

Поступила в редакцию 06.06.2021 г.

В окончательной редакции 23.08.2021 г.

Принята к публикации 02.09.2021 г.

\begin{abstract}
Выявлены математические свойства функций, характеризующих симметрии пространственного распределения излучения второй гармоники-суммарной частоты. Установлены условия, при которых генерация излучения не происходит, и условия, при которых генерируемое излучение имеет линейную поляризацию. Обнаруженные свойства систематизированы по их проявлениям на диаграммах направленности генерируемого излучения. Предложены методы определения компонент тензора нелинейной диэлектрической восприимчивости на основе указанных свойств. Описана связь обнаруженных свойств и условий с ранее найденными аналогичными свойствами и условиями для явлений генерации второй гармоники и генерации суммарной частоты.
\end{abstract}

Ключевые слова: генерация второй гармоники, генерация суммарной частоты, диэлектрическая сферическая частица, поляризация, свойства диаграммы направленности.

DOI: $10.21883 / \mathrm{OS} .2021 .12 .51744 .2387-21$

\section{Свойства функций, характеризующих пространственное распределение генерируемого излучения}

Анализ функций, характеризующих пространственное распределение излучения второй гармоники-суммарной частоты, позволяет обнаружить у них некоторые математические свойства. Они характеризуют соотношения между компонентами вектора электрической напряжённости генерируемого излучения, а также симметрии его пространственного распределения. Аналогичные свойства ранее были обнаружены для нелинейной генерации в тонком слое на поверхности сферических $[1,2]$ и цилиндрических частиц [3-5].

В случае сонаправленного распространения падающих электромагнитных волн, имеющих любые амплитуды и поляризации, функции $\mathbf{f}^{(2 \omega)}(\theta, \varphi)$ и $S_{r}^{(2 \omega)}(\theta, \varphi)$, характеризующие пространственное распределение генерируемого излучения, совпадают с аналогичными функциями в задаче о генерации второй гармоники [1] при эквивалентных значениях параметров задачи, описанных в части I (формулы (29)-(30)). Поэтому далее в работе не рассматривается случай сонаправленного распространения падающих электромагнитных волн. Свойства для этого случая с учётом замены (30) части I можно получить из свойств в работе [1], посвящённой генерации второй гармоники в тонком оптически нелинейном сферическом слое.

В табл. 1 приведены математические свойства функций $\mathbf{f}^{(2 \omega)}(\theta, \varphi)$ и $S_{r}^{(2 \omega)}(\theta, \varphi)$, а также комбинации параметров задачи и накладываемых на них ограничений, при которых эти свойства проявляются. Символ $\forall$ в таблице означает, что следующая за ним величина может принимать любое действительное значение. Переменные $m, m_{1}, m_{2}$ в таблице означают целые числа, подобранные таким образом, чтобы параметры задачи находились в следующих пределах:

$$
\begin{gathered}
-\pi<\varphi_{\text {in }}^{(1)} \leq \pi, \quad-\pi<\varphi_{\text {in }}^{(2)} \leq \pi, \quad 0 \leq \theta \leq \pi \\
-\pi<\varphi \leq \pi, \quad-1 \leq \sigma_{1} \leq 1, \quad-1 \leq \sigma_{2} \leq 1 \\
0 \leq \gamma_{1} \leq \pi, \quad 0 \leq \gamma_{2} \leq \pi
\end{gathered}
$$

Параметр $\eta$ в задаче характеризует отношение амплитуд напряжённостей падающих волн, а также разность их фаз, поэтому он может принимать как комплексные значения $(\eta \in \mathbb{C})$, так и действительные $(\operatorname{Im}[\eta]=0)$. Подразумевается, что коэффициенты $\chi_{1-4}^{(2)}$ принимают только действительные значения. Если условие для какого-то параметра задачи не задано, то подразумевается, что этот параметр может принимать любое действительное значение. Рассмотрим отдельные свойства в качестве примера.

В соответствии со строкой 4 табл. 1 при падении плоских электромагнитных волн, имеющих произвольную поляризацию (не указаны ограничения на $\sigma_{1}, \sigma_{2}, \varphi_{\text {in }}^{(1)}$, $\left.\varphi_{\text {in }}^{(2)}\right)$, амплитуду $(\eta \in \mathbb{C})$ и произвольные направления волновых векторов (не указаны ограничения на $\gamma_{1}, \gamma_{2}$ ), на сферический оптически нелинейных слой, в тензоре нелинейной диэлектрической восприимчивости которого коэффициент $\chi_{2}^{(2)}$ принимает любые значения $\left(\forall \chi_{2}^{(2)}\right)$, а остальные коэффициенты $\chi_{1,3,4}^{(2)}$ равны нулю $\left(\chi_{1,3,4}^{(2)}=0\right)$, 
пространственное распределение плотности мощности генерируемого излучения имеет зеркальную плоскость симметрии $O y z\left(S_{r}^{(2 \omega)}(\theta, \pi-\varphi)=S_{r}^{(2 \omega)}(\theta, \varphi)\right)$, содержащую волновые векторы падающих волн (если не выполняется условие $\gamma_{1}+\gamma_{2}=\pi m$, где $m-$ целое число). Следствием этого является равенство нулю асимметрии $A_{\mathrm{UD}}$, что наглядно видно на рис. $4, c, 5, c, 6, c, 7, c$ части II.

Для строки 1 проявление зеркальных плоскостей симметрий диаграммы направленности можно видеть на графике асимметрии $A_{\mathrm{UD}}\left(\varphi_{\mathrm{in}}^{(2)}\right)$ (рис. 6, $c$ части II), где данное условие выполняется в точках $\varphi_{\text {in }}^{(2)}= \pm \pi / 2$. При этом данные асимметрии для всех типов анизотропии обращаются в ноль.

В соответствии с информацией, содержащейся в строке 16 табл. 1, при встречном падении $\left(\gamma_{1}=0, \gamma_{2}=\pi\right)$ двух плоских электромагнитных волн, имеющих левую и правую циркулярную поляризации $\left(\sigma_{1} \sigma_{2}=-1\right)$ и произвольную амплитуду и фазу $(\eta \in \mathbb{C})$, на тонкий сферический оптически нелинейный слой, имеющий любые действительные значения коэффициентов анизотропии $\left(\forall \chi_{1-4}^{(2)}\right)$, пространственное распределение плотности мощности генерируемого излучения имеет аксиальную симметрию относительно оси $\mathrm{Oz}$ $\left(S_{r}^{(2 \omega)}(\theta, \varphi+\Delta \varphi)=S_{r}^{(2 \omega)}(\theta, \varphi)\right)$. Подобная конфигурация падающих электромагнитных волн, но с близкими амплитудами, может быть использована для получения более интенсивного отклика киральных молекул по сравнению со случаем облучения киральных молекул одиночной циркулярно поляризованной волной [6]. Аналогично для изучения поверхностных слоёв может применяться и схема со встречными линейно поляризованными волнами [7].

Сведения в строке 24 табл. 1 можно трактовать следующим образом: при встречном падении $\left(\gamma_{1}=0\right.$, $\left.\gamma_{2}=\pi\right)$ двух плоских электромагнитных волн, имеющих произвольную поляризацию, амплитуду и фазу, на тонкий оптически нелинейный киральный слой, имеющий равные нулю коэффициенты анизотропии $\chi_{1,2,4}^{(2)}$, в плоскости Oxy $(\theta=\pi / 2)$ генерируется излучение, которое не имеет составляющей вектора напряженности электрического поля вдоль вектора $\mathbf{e}_{\theta}\left(\mathbf{e}_{\theta} \mathbf{f}^{(2 \omega)}(\theta, \varphi)=0\right)$.

Приведенные в табл. 1 свойства можно сгруппировать по проявлениям на диаграммах направленности, характеризующих пространственное распределение плотности мощности генерируемого излучения.

1) Зеркальная симметрия диаграммы направленности относительно плоскости $O y z$ : строки 1, 4. Для неё характерно математическое свойство

$$
S_{r}^{(2 \omega)}(\theta, \pi-\varphi)=S_{r}^{(2 \omega)}(\theta, \varphi)
$$

2) Зеркальная симметрия диаграммы направленности относительно плоскости $O x z$ : строки 9, 10, 12, 14, 17. Для неё характерно математическое свойство

$$
S_{r}^{(2 \omega)}(\theta,-\varphi)=S_{r}^{(2 \omega)}(\theta, \varphi) .
$$

3) Поворотная ось симметрии второго порядка $O z$ : строки 8 (её можно видеть на рис. $3, d, 8, d$ части II), $11,13,15$. Для такой оси характерно математическое свойство

$$
S_{r}^{(2 \omega)}(\theta, \varphi+\pi)=S_{r}^{(2 \omega)}(\theta, \varphi) .
$$

4) Аксиальная симметрия относительно оси $O z$ : строки 16,18 . Для неё характерно математическое свойство

$$
S_{r}^{(2 \omega)}(\theta, \varphi+\Delta \varphi)=S_{r}^{(2 \omega)}(\theta, \varphi), \quad \forall \Delta \varphi .
$$

5) Отсутствие составляющей вектора электрической напряжённости генерируемого поля вдоль вектора $\mathbf{e}_{\theta}$ : строки 24, 27.

6) Отсутствие составляющей вектора электрической напряжённости генерируемого поля вдоль вектора $\mathbf{e}_{\varphi}$ : строки 25, 26, 28.

7) Составляющие вектора электрической напряжённости генерируемого излучения вдоль векторов $\mathbf{e}_{\theta}$ и $\mathbf{e}_{\varphi}$ имеют одинаковую фазу: строки 2, 3, 6, 7, 19, 20.

8) Поворотная ось симметрии четвёртого порядка для модуля составляющей вектора электрической напряжённости генерируемого излучения, направленной вдоль $\mathbf{e}_{\varphi}$ : строка 21.

9) Поворотная ось симметрии четвёртого порядка для модуля составляющей вектора электрической напряжённости генерируемого излучения, направленной вдоль $\mathbf{e}_{\theta}$ : строка 22.

10) В строках 5 и 23 приведены свойства, относящиеся к симметриям действительной части функции $\mathbf{f}^{(2 \omega)}(\theta, \varphi)$, которые нельзя выявить посредством анализа диаграмм направленности.

Отдельно стоит сказать про математические свойства в строке 29 табл. 1. Эти свойства были замечены ранее по отдельности для генерации суммарной частоты в нелинейном слое на поверхности сферической [2] и цилиндрической [5] частиц, а также для генерации второй гармоники в нелинейном слое на поверхности сферической [1] и цилиндрической [3] частиц. Они не имеют явного физического смысла и являются следствием математических свойств периодических функций углов $\theta$ и $\varphi$, входящих в состав $\mathbf{f}^{(2 \omega)}(\theta, \varphi)$ и $S_{r}^{(2 \omega)}(\theta, \varphi)$.

\section{Условия отсутствия генерации}

При некоторых значениях параметров задачи генерация второй гармоники-суммарной частоты отсутствует вовсе или не наблюдается в определённых направлениях. Такие комбинации параметров задачи можно использовать для оценки коэффициентов анизотропии нелинейного слоя. Подобный метод, например, предложен при рассмотрении генерации второй гармоники в нелинейном слое на поверхности сферической [1] или цилиндрической [3] частицы.

Условия отсутствия генерации второй гармоникисуммарной частоты в нелинейном слое на поверхности сферической частицы приведены в табл. 2. Обозначения 
Таблица 1. Математические свойства функций, характеризующих пространственное распределение генерируемого излучения

\begin{tabular}{|c|c|c|}
\hline № & Комбинации параметров задачи & Свойства \\
\hline 1 & $\begin{array}{c}\forall \chi_{1-4}^{(2)}, \operatorname{Im}[\eta]=0 \\
\varphi_{\text {in }}^{(1)}=\pi m_{1} / 2 \\
\varphi_{\text {in }}^{(2)}=\pi m_{1} / 2+\pi m_{2}\end{array}$ & $\begin{array}{c}i\left(1-(1-i) \mathbf{e}_{\varphi} \otimes \mathbf{e}_{\varphi}\right) \mathbf{f}^{(2 \omega)}(\theta, \pi-\varphi)= \\
=\left[i\left(1-(1-i) \mathbf{e}_{\varphi} \otimes \mathbf{e}_{\varphi}\right) \mathbf{f}^{(2 \omega)}(\theta, \varphi)\right]^{*}, \\
i\left(1-(1-i) \mathbf{e}_{\theta} \otimes \mathbf{e}_{\theta}\right) \mathbf{f}^{(2 \omega)}(-\theta, \varphi)= \\
=\left[i\left(1-(1-i) \mathbf{e}_{\theta} \otimes \mathbf{e}_{\theta}\right) \mathbf{f}^{(2 \omega)}(\theta,-\varphi)\right]^{*} \\
S_{r}^{(2 \omega)}(\theta, \pi-\varphi)=S_{r}^{(2 \omega)}(\theta, \varphi)\end{array}$ \\
\hline 2 & $\begin{array}{c}\forall \chi_{1-3}^{(2)}, \chi_{4}^{(2)}=0 \\
\operatorname{Im}[\eta]=0, \sigma_{1}=\sigma_{2}=0\end{array}$ & $\operatorname{Re}\left[\mathbf{f}^{(2 \omega)}(\theta, \varphi)\right]=0$ \\
\hline 3 & $\begin{array}{c}\forall \chi_{4}^{(2)}, \chi_{1-3}^{(2)}=0, \operatorname{Im}[\eta]=0 \\
\sigma_{1}=\sigma_{2}=0\end{array}$ & $\operatorname{Im}\left[\mathbf{f}^{(2 \omega)}(\theta, \varphi)\right]=0$ \\
\hline 4 & $\forall \chi_{2}^{(2)}, \chi_{1,3,4}^{(2)}=0, \eta \in \mathbb{C}$ & $\begin{array}{c}\mathbf{f}^{(2 \omega)}(\theta, \pi-\varphi)=\left(1-2 \mathbf{e}_{\varphi} \otimes \mathbf{e}_{\varphi}\right) \mathbf{f}^{(2 \omega)}(\theta, \varphi), \\
\mathbf{f}^{(2 \omega)}(-\theta, \varphi)=\left(1-2 \mathbf{e}_{\theta} \otimes \mathbf{e}_{\theta}\right) \mathbf{f}^{(2 \omega)}(\theta,-\varphi), \\
S_{r}^{(2 \omega)}(\theta, \pi-\varphi)=S_{r}^{(2 \omega)}(\theta, \varphi), \\
S_{r}^{(2 \omega)}(-\theta, \varphi)=S_{r}^{(2 \omega)}(\theta,-\varphi)\end{array}$ \\
\hline 5 & $\begin{array}{c}\forall \chi_{2}^{(2)}, \chi_{1,3,4}^{(2)}=0 \\
\arg (\eta)=m_{1} \pi / 2 \\
\forall \Delta \varphi\end{array}$ & $\begin{array}{c}\operatorname{Re}\left[\mathbf{e}_{\varphi} \mathbf{f}^{(2 \omega)}(\theta, \varphi)\right]=0 \\
\operatorname{Re}\left[\mathbf{f}^{(2 \omega)}(\theta, \varphi+\Delta \varphi)\right]=\operatorname{Re}\left[\mathbf{f}^{(2 \omega)}(\theta, \varphi)\right], \\
\operatorname{Re}\left[\mathbf{f}^{(\omega)}(-\theta, \varphi)\right]= \\
=\operatorname{Re}\left[\left(1-2 \mathbf{e}_{\theta} \otimes \mathbf{e}_{\theta}\right) \mathbf{f}^{(2 \omega)}(\theta, \varphi)\right]\end{array}$ \\
\hline 6 & $\begin{array}{c}\forall \chi_{2}^{(2)}, \chi_{1,3,4}^{(2)}=0, \operatorname{Im}[\eta]=0, \\
\varphi_{\mathrm{in}}^{(1)}=\pi m_{1} / 2, \varphi_{\mathrm{in}}^{(2)}=\pi m_{1} / 2+\pi m_{2}\end{array}$ & $\operatorname{Re}\left[\mathbf{f}^{(2 \omega)}(\theta, \varphi)\right]=0$ \\
\hline 7 & $\begin{array}{c}\forall \chi_{2}^{(2)}, \chi_{1,3,4}^{(2)}=0, \operatorname{Im}[\eta]=0, \\
\sigma_{1}=(-1)^{m_{1}} \sigma_{2} \\
\varphi_{\mathrm{in}}^{(1)}+(-1)^{m_{1}} \varphi_{\mathrm{in}}^{(2)}=\pi m_{2}\end{array}$ & $\operatorname{Re}\left[\mathbf{f}^{(2 \omega)}(\theta, \varphi)\right]=0$ \\
\hline 8 & $\begin{array}{c}\forall \chi_{1-4}^{(2)}, \eta= \pm 1 \\
\sigma_{1}=\sigma_{2}, \varphi_{\text {in }}^{(1)}-\varphi_{\text {in }}^{(2)}=\pi m_{1}\end{array}$ & $\begin{array}{l}\mathbf{f}^{(2 \omega)}(\theta, \varphi+\pi)=\mathbf{f}^{(2 \omega)}(\theta, \varphi), \\
S_{r}^{(2 \omega)}(\theta, \varphi+\pi)=S_{r}^{(2 \omega)}(\theta, \varphi)\end{array}$ \\
\hline 9 & $\begin{array}{c}\forall \chi_{1-4}^{(2)},|\eta|=1 \\
\sigma_{1}=\sigma_{2}, \varphi_{\mathrm{in}}^{(1)}+\varphi_{\mathrm{in}}^{(2)}=\pi m_{1}\end{array}$ & $\begin{array}{c}i\left(1-(1-i) \mathbf{e}_{\varphi} \otimes \mathbf{e}_{\varphi}\right) \mathbf{f}^{(2 \omega)}(\theta,-\varphi) / \eta= \\
=\left[i(1-i) \mathbf{e}_{\varphi} \otimes \mathbf{e}_{\varphi} \mathbf{f}^{(2 \omega)}(\theta, \varphi) / \eta\right]^{*} \\
S_{r}^{(2 \omega)}(\theta,-\varphi)=S_{r}^{(2 \omega)}(\theta, \varphi)\end{array}$ \\
\hline 10 & $\begin{array}{c}\forall \chi_{1-3}^{(2)}, \chi_{4}^{(2)}=0, \eta= \pm 1 \\
\sigma_{1}=-\sigma_{2}, \varphi_{\mathrm{in}}^{(1)}+\varphi_{\mathrm{in}}^{(2)}=\pi m_{1}\end{array}$ & $\begin{array}{c}\mathbf{f}^{(2 \omega)}(\theta,-\varphi)=\left(1-2 \mathbf{e}_{\varphi} \otimes \mathbf{e}_{\varphi}\right) \mathbf{f}^{(2 \omega)}(\theta, \varphi) \\
S_{r}^{(2 \omega)}(\theta,-\varphi)=S_{r}^{(2 \omega)}(\theta, \varphi)\end{array}$ \\
\hline 11 & $\begin{array}{c}\forall \chi_{1-3}^{(2)}, \chi_{4}^{(2)}=0,|\eta|=1 \\
\sigma_{1}=-\sigma_{2}, \varphi_{\mathrm{in}}^{(1)}-\varphi_{\mathrm{in}}^{(2)}=\pi m_{1}\end{array}$ & $\begin{array}{c}\mathbf{f}^{(2 \omega)}(\theta, \varphi+\pi) / \eta=-\left[\mathbf{f}^{(2 \omega)}(\theta, \varphi) / \eta\right]^{*} \\
S_{r}^{(2 \omega)}(\theta, \varphi+\pi)=S_{r}^{(2 \omega)}(\theta, \varphi)\end{array}$ \\
\hline 12 & $\begin{array}{c}\forall \chi_{4}^{(2)}, \chi_{1-3}^{(2)}=0, \eta= \pm 1 \\
\sigma_{1}=-\sigma_{2}, \varphi_{\text {in }}^{(1)}+\varphi_{\text {in }}^{(2)}=\pi m_{1}\end{array}$ & $\begin{array}{c}\mathbf{f}^{(2 \omega)}(\theta,-\varphi)=-\left(1-2 \mathbf{e}_{\varphi} \otimes \mathbf{e}_{\varphi}\right) \mathbf{f}^{(2 \omega)}(\theta, \varphi) \\
S_{r}^{(2 \omega)}(\theta,-\varphi)=S_{r}^{(2 \omega)}(\theta, \varphi)\end{array}$ \\
\hline 13 & $\begin{array}{c}\forall \chi_{4}^{(2)}, \chi_{1-3}^{(2)}=0,|\eta|=1 \\
\sigma_{1}=-\sigma_{2}, \varphi_{\mathrm{in}}^{(1)}-\varphi_{\mathrm{in}}^{(2)}=\pi m_{1}\end{array}$ & $\begin{array}{c}\mathbf{f}^{(2 \omega)}(\theta, \varphi+\pi) / \eta=\left[\mathbf{f}^{(2 \omega)}(\theta, \varphi) / \eta\right]^{*} \\
S_{r}^{(2 \omega)}(\theta, \varphi+\pi)=S_{r}^{(2 \omega)}(\theta, \varphi)\end{array}$ \\
\hline 14 & $\begin{array}{c}\forall \chi_{2}^{(2)}, \chi_{1,3,4}^{(2)}=0, \eta= \pm 1 \\
\sigma_{1}=(-1)^{m} \sigma_{2}\end{array}$ & $\begin{array}{c}\mathbf{f}^{(2 \omega)}(\theta,-\varphi)=\left(1-2 \mathbf{e}_{\varphi} \otimes \mathbf{e}_{\varphi}\right) \mathbf{f}^{(2 \omega)}(\theta, \varphi) \\
S_{r}^{(2 \omega)}(\theta,-\varphi)=S_{r}^{(2 \omega)}(\theta, \varphi)\end{array}$ \\
\hline 15 & $\begin{array}{c}\forall \chi_{1-4}^{(2)}, \eta \in \mathbb{C} \\
\gamma_{1}=0, \gamma_{2}=\pi\end{array}$ & $\begin{array}{l}\mathbf{f}^{(2 \omega)}(\theta, \varphi+\pi)=\mathbf{f}^{(2 \omega)}(\theta, \varphi), \\
S_{r}^{(2 \omega)}(\theta, \varphi+\pi)=S_{r}^{(2 \omega)}(\theta, \varphi)\end{array}$ \\
\hline 16 & $\begin{array}{c}\forall \chi_{1-4}^{(2)}, \eta \in \mathbb{C} \\
\gamma_{1}=0, \gamma_{2}=\pi, \sigma_{1} \sigma_{2}=-1\end{array}$ & $\begin{array}{c}\mathbf{f}^{(2 \omega)}(\theta, \varphi+\Delta \varphi)= \\
=\mathbf{f}^{(2 \omega)}(\theta, \varphi) \exp \left(i\left(\sigma_{1}-\sigma_{2}\right) \Delta \varphi\right), \\
S_{r}^{(2 \omega)}(\theta, \varphi+\Delta \varphi)=S_{r}^{(2 \omega)}(\theta, \varphi)\end{array}$ \\
\hline 17 & $\begin{array}{c}\forall \chi_{1-4}^{(2)}, \operatorname{Im}[\eta]=0 \\
\gamma_{1}=0, \gamma_{2}=\pi, \\
\varphi_{\text {in }}^{(1)}=m_{1} \pi / 2, \varphi_{\text {in }}^{(2)}=m_{1} \pi / 2+\pi m_{2}\end{array}$ & $\begin{array}{c}i\left(1-(1-i) \mathbf{e}_{\varphi} \otimes \mathbf{e}_{\varphi}\right) \mathbf{f}^{(2 \omega)}(\theta,-\varphi)= \\
=\left[i\left(1-(1-i) \mathbf{e}_{\varphi} \otimes \mathbf{e}_{\varphi}\right) \mathbf{f}^{(2 \omega)}(\theta, \varphi)\right]^{*} \\
S_{r}^{(2 \omega)}(\theta,-\varphi)=S_{r}^{(2 \omega)}(\theta, \varphi)\end{array}$ \\
\hline
\end{tabular}


Таблица. 1 (продолжение)

\begin{tabular}{|c|c|c|}
\hline № & Комбинации параметров задачи & Свойства \\
\hline 18 & $\begin{array}{c}\forall \chi_{2}^{(2)}, \chi_{1,3,4}^{(2)}=0, \eta \in \mathbb{C} \\
\gamma_{1}=0, \gamma_{2}=\pi\end{array}$ & $\begin{array}{c}\mathbf{e}_{\varphi} \mathbf{f}^{(2 \omega)}(\theta, \varphi)=0, \\
\mathbf{f}^{(2 \omega)}(\theta, \varphi+\Delta \varphi)=\mathbf{f}^{(2 \omega)}(\theta, \varphi), \\
S_{r}^{(2 \omega)}(\theta, \varphi+\Delta \varphi)=S_{r}^{(2 \omega)}(\theta, \varphi)\end{array}$ \\
\hline 19 & $\begin{array}{c}\forall \chi_{2}^{(2)}, \chi_{1,3,4}^{(2)}=0, \operatorname{Im}[\eta]=0 \\
\sigma_{1}=-\sigma_{2}, \gamma_{1}=0, \gamma_{2}=\pi\end{array}$ & $\operatorname{Re}\left[\mathbf{f}^{(2 \omega)}(\theta, \varphi)\right]=0$ \\
\hline 20 & $\begin{aligned} \forall \chi_{2}^{(2)}, \chi_{1,3,4}^{(2)} & =0, \arg (\eta)=m \pi / 2 \\
\gamma_{1} & =0, \gamma_{2}=\pi\end{aligned}$ & $\operatorname{Re}\left[\left(1-\mathbf{e}_{r} \otimes \mathbf{e}_{r}\right) \mathbf{f}^{(2 \omega)}(\theta, \varphi)\right]=0$ \\
\hline 21 & $\begin{array}{c}\forall \chi_{1-3}^{(2)}, \chi_{4}^{(2)}=0, \eta \in \mathbb{C} \\
\gamma_{1}=0, \gamma_{2}=\pi\end{array}$ & $\mathbf{e}_{\varphi} \mathbf{f}^{(2 \omega)}\left(\theta, \varphi+m \frac{\pi}{2}\right)=(-1)^{m} \mathbf{e}_{\varphi} \mathbf{f}^{(2 \omega)}(\theta, \varphi)$ \\
\hline 22 & $\begin{array}{c}\forall \chi_{4}^{(2)}, \chi_{1-3}^{(2)}=0, \eta \in \mathbb{C} \\
\gamma_{1}=0, \gamma_{2}=\pi\end{array}$ & $\begin{array}{c}\left(1-\mathbf{e}_{\varphi} \otimes \mathbf{e}_{\varphi}\right) \mathbf{f}^{(2 \omega)}(\theta, \varphi+m \pi / 2)= \\
=(-1)^{m}\left(1-\mathbf{e}_{\varphi} \otimes \mathbf{e}_{\varphi}\right) \mathbf{f}^{(2 \omega)}(\theta, \varphi)\end{array}$ \\
\hline 23 & $\begin{array}{l}\forall \chi_{1-3}^{(2)}, \chi_{4}^{(2)}=0, \operatorname{Im}[\eta]=0 \\
\gamma_{1}=0, \gamma_{2}=\pi, \sigma_{1}=-\sigma_{2}\end{array}$ & $\begin{array}{l}\operatorname{Re}\left[\mathbf{f}^{(2 \omega)}(\theta, \varphi+m \pi / 2)\right]= \\
=(-1)^{m} \operatorname{Re}\left[\mathbf{f}^{(2 \omega)}(\theta, \varphi)\right]\end{array}$ \\
\hline 24 & $\begin{array}{c}\forall \chi_{3}^{(2)}, \chi_{1,2,4}^{(2)}=0, \eta \in \mathbb{C} \\
\gamma_{1}=0, \gamma_{2}=\pi, \theta=\pi / 2\end{array}$ & $\mathbf{e}_{\theta} \mathbf{f}^{(2 \omega)}(\theta, \varphi)=0$ \\
\hline 25 & $\begin{array}{c}\forall \chi_{1-4}^{(2)}, \eta \in \mathbb{C} \\
\gamma_{1}=0, \gamma_{2}=\pi, \sigma_{1}=\sigma_{2}=0, \\
\varphi_{\text {in }}^{(1)}+\varphi_{\text {in }}^{(2)}=\pi m, \varphi= \pm \pi / 2+\varphi_{\text {in }}^{(1)}\end{array}$ & $\mathbf{e}_{\varphi} \mathbf{f}^{(2 \omega)}(\theta, \varphi)=0$ \\
\hline 26 & $\begin{array}{c}\forall \chi_{1-3}^{(2)}, \chi_{4}^{(2)}=0, \eta \in \mathbb{C} \\
\gamma_{1}=0, \gamma_{2}=\pi, \sigma_{1}=\sigma_{2}=0 \\
\varphi_{\mathrm{in}}^{(1)}+\varphi_{\mathrm{in}}^{(2)}=\pi m_{1}, \varphi=\varphi_{\mathrm{in}}^{(1)}+\pi m_{2}\end{array}$ & $\mathbf{e}_{\varphi} \mathbf{f}^{(2 \omega)}(\theta, \varphi)=0$ \\
\hline 27 & $\begin{array}{c}\forall \chi_{4}^{(2)}, \chi_{1-3}^{(2)}=0, \eta \in \mathbb{C} \\
\gamma_{1}=0, \gamma_{2}=\pi, \sigma_{1}=\sigma_{2}=0 \\
\varphi_{\mathrm{in}}^{(1)}+\varphi_{\mathrm{in}}^{(2)}=\pi m_{1}, \varphi=\varphi_{\mathrm{in}}^{(1)}+\pi m_{2}\end{array}$ & $\mathbf{e}_{\theta} \mathbf{f}^{(2 \omega)}(\theta, \varphi)=0$ \\
\hline 28 & $\begin{array}{c}\forall \chi_{2,4}^{(2)}, \chi_{1,3}^{(2)}=0, \eta= \pm 1 \\
\gamma_{1}=0, \gamma_{2}=\pi, \sigma_{1}=-\sigma_{2} \\
\varphi_{\mathrm{in}}^{(1)}+\varphi_{\mathrm{in}}^{(2)}=\pi m, \theta=\pi / 2\end{array}$ & $\mathbf{e}_{\varphi} \mathbf{f}^{(2 \omega)}(\theta, \varphi)=0$ \\
\hline 29 & $\forall \chi_{1-4}^{(2)}, \eta \in \mathbb{C}$ & $\begin{array}{c}\mathbf{f}^{(2 \omega)}\left(\theta+2 \pi m_{1}, \varphi+2 \pi m_{2}\right)=\mathbf{f}^{(2 \omega)}(\theta, \varphi), \\
\mathbf{f}^{(2 \omega)}(-\theta, \varphi)=-\left(1-2 \mathbf{e}_{r} \otimes \mathbf{e}_{r}\right) \mathbf{f}^{(2 \omega)}(\theta, \varphi+\pi), \\
S_{r}^{(2 \omega)}\left(\theta+2 \pi m_{1}, \varphi+2 \pi m_{2}\right)=S_{r}^{(2 \omega)}(\theta, \varphi), \\
S_{r}^{(2 \omega)}(-\theta, \varphi)=S_{r}^{(2 \omega)}(\theta, \varphi+\pi)\end{array}$ \\
\hline
\end{tabular}

для записи условий во втором столбце табл. 2 совпадают с обозначениями, принятыми для табл. 1. В третьем столбце табл. 2 приведены функции, которые обращаются в ноль при соблюдении условий из второго столбца табл. 2. В табл. 2 также используется обозначение $Z_{1}^{(j)}-$ ноль функции Бесселя первого порядка:

$$
j_{1}\left(Z_{1}^{(j)}\right)=0 .
$$

Рассмотрим некоторые из строк.

В строке 3 табл. 2 указано, что при встречном падении двух плоских электромагнитных волн $\left(\gamma_{1}=0, \gamma_{2}=\pi\right)$, имеющих произвольную поляризацию, амплитуду и фазу, на нелинейный сферический слой, имеющий произвольные значения коэффициентов анизотропии $\left(\forall \chi_{1-4}^{(2)}\right)$, в направлениях, параллельных оси $O z$, излучение не генерируется. Похожее свойство уже описано в предыдущих работах по теме нелинейной генерации $[1,3,8]$ для генерации второй гармоники плоской электромагнитной волной в нелинейном слое на поверхности сферической диэлектрической частицы: генерация не наблюдается вдоль прямой, содержащей волновой вектор падающей волны. Аналогичное свойство можно наблюдать и при генерации суммарной частоты: при встречном падении двух плоских электромагнитных волн, имеющих произвольные частоты, амплитуды, поляризации и фазы, генерация суммарной частоты не наблюдается вдоль направлений их волновых векторов, что дополняет работу [2]. 
Таблица 2. Комбинации значений параметров задачи, при которых генерация отсутствует

\begin{tabular}{|c|c|c|}
\hline № & Комбинации параметров задачи & Свойства \\
\hline 1 & $\begin{array}{c}\forall \chi_{2}^{(2)}, \chi_{1,3,4}^{(2)}=0, \eta \in \mathbb{C} \\
\sigma_{1} \sigma_{2}=1, \gamma_{1}=0, \gamma_{2}=\pi\end{array}$ & $\begin{array}{c}\left(1-\mathbf{e}_{r} \otimes \mathbf{e}_{r}\right) \mathbf{f}^{(2 \omega)}(\theta, \varphi)=0, \\
S_{r}^{(2 \omega)}(\theta, \varphi)=0\end{array}$ \\
\hline 2 & $\begin{array}{c}\forall \chi_{2}^{(2)}, \chi_{1,3,4}^{(2)}=0, \eta \in \mathbb{C} \\
\sigma_{1} \sigma_{2}=-1, \gamma_{1}=0, \gamma_{2}=\pi\end{array}$ & $\begin{array}{l}\mathbf{f}^{(2 \omega)}(\theta, \varphi)=0 \\
S_{r}^{(2 \omega)}(\theta, \varphi)=0\end{array}$ \\
\hline 3 & $\begin{array}{c}\forall \chi_{1-4}^{(2)}, \eta \in \mathbb{C} \\
\gamma_{1}=0, \gamma_{2}=\pi, \theta=0, \pi\end{array}$ & $\begin{array}{l}\mathbf{f}^{(2 \omega)}(\theta, \varphi)=0 \\
S_{r}^{(2 \omega)}(\theta, \varphi)=0\end{array}$ \\
\hline 4 & $\begin{array}{c}\forall \chi_{2}^{(2)}, \chi_{1,3,4}^{(2)}=0, \eta \in \mathbb{C} \\
\gamma_{1}=0, \gamma_{2}=\pi, \sigma_{1}=-\sigma_{2} \\
\varphi_{\mathrm{in}}^{(1)}+\varphi_{\mathrm{in}}^{(2)}=\pi m, q^{(11)} a=Z_{1}^{(j)}\end{array}$ & $\begin{array}{c}\left(1-\mathbf{e}_{r} \otimes \mathbf{e}_{r}\right) \mathbf{f}^{(12)}(\theta, \varphi)=0, \\
\mathbf{f}^{(11)}(\theta, \varphi)=0 \\
S_{r}^{(2 \omega)}(\theta, \varphi)=S_{r}^{(22)}(\theta, \varphi)\end{array}$ \\
\hline 5 & $\begin{array}{c}\forall \chi_{2}^{(2)}, \chi_{1,3,4}^{(2)}=0, \eta \in \mathbb{C} \\
\gamma_{1}=0, \gamma_{2}=\pi, \sigma_{1}=-\sigma_{2} \\
\varphi_{\text {in }}^{(1)}+\varphi_{\text {in }}^{(2)}=\pi m, q^{(22)} a=Z_{1}^{(j)}\end{array}$ & $\begin{array}{c}\left(1-\mathbf{e}_{r} \otimes \mathbf{e}_{r}\right) \mathbf{f}^{(12)}(\theta, \varphi)=0 \\
\mathbf{f}^{(22)}(\theta, \varphi)=0 \\
S_{r}^{(2 \omega)}(\theta, \varphi)=S_{r}^{(11)}(\theta, \varphi)\end{array}$ \\
\hline 6 & $\begin{array}{c}\forall \chi_{2}^{(2)}, \chi_{1,3,4}^{(2)}=0, \arg \eta=m_{1} \pi / 2 \\
\gamma_{1}=0, \gamma_{2}=\pi, \sigma_{1}=-\sigma_{2}, \varphi_{\text {in }}^{(1)}+\varphi_{\text {in }}^{(2)}=\pi m \\
\frac{j_{1}\left(q^{(11)}(\theta, \varphi) a\right)}{q^{(11)}}=\eta^{2} \frac{j_{1}\left(q^{(2)}(\theta, \varphi) a\right)}{q^{(22)}}\end{array}$ & $\begin{array}{c}\left(1-\mathbf{e}_{r} \otimes \mathbf{e}_{r}\right) \mathbf{f}^{(2 \omega)}(\theta, \varphi)=0 \\
S_{r}^{(2 \omega)}(\theta, \varphi)=0\end{array}$ \\
\hline 7 & $\begin{array}{c}\forall \chi_{2}^{(2)}, \chi_{1,3,4}^{(2)}=0, \eta \in \mathbb{C}, \gamma_{1}=0, \gamma_{2}=\pi, \sigma_{1}=-\sigma_{2}, \\
\varphi_{\mathrm{in}}^{(1)}+\varphi_{\mathrm{in}}^{(2)}=\pi m, \theta=\pi / 2, j_{1}\left(q^{(11)} a\right)=j_{1}\left(q^{(22)} a\right)=0\end{array}$ & $\begin{array}{c}\left(1-\mathbf{e}_{r} \otimes \mathbf{e}_{r}\right) \mathbf{f}^{(2 \omega)}(\theta, \varphi)=0 \\
S_{r}^{(2 \omega)}(\theta, \varphi)=0\end{array}$ \\
\hline 8 & $\begin{array}{c}\forall \chi_{2}^{(2)}, \chi_{1,3,4}^{(2)}=0, \eta= \pm 1 \\
\gamma_{1}=0, \gamma_{2}=\pi, \sigma_{1}=-\sigma_{2}, \theta=\pi / 2\end{array}$ & $\begin{array}{c}\left(1-\mathbf{e}_{r} \otimes \mathbf{e}_{r}\right) \mathbf{f}^{(2 \omega)}(\theta, \varphi)=0 \\
S_{r}^{(2 \omega)}(\theta, \varphi)=0\end{array}$ \\
\hline 9 & $\begin{array}{c}\forall \chi_{3,4}^{(2)}, \chi_{1,2}^{(2)}=0, \eta \in \mathbb{C}, \gamma_{1}=0, \gamma_{2}=\pi, \sigma_{1}=\sigma_{2}=0, \\
\varphi_{\mathrm{in}}^{(1)}+\varphi_{\mathrm{in}}^{(2)}=\pi m_{1}, \varphi=\varphi_{\mathrm{in}}^{(1)}+\pi / 2+\pi m_{2}\end{array}$ & $\begin{array}{l}\mathbf{f}^{(2 \omega)}(\theta, \varphi)=0 \\
S_{r}^{(2 \omega)}(\theta, \varphi)=0\end{array}$ \\
\hline 10 & $\begin{array}{c}\forall \chi_{3}^{(2)}, \chi_{1,2,4}^{(2)}=0, \eta \in \mathbb{C}, \gamma_{1}=0, \gamma_{2}=\pi, \sigma_{1}=\sigma_{2}=0 \\
\varphi_{\mathrm{in}}^{(1)}+\varphi_{\mathrm{in}}^{(2)}=\pi m_{1}, \theta=\pi / 2, \varphi=\varphi_{\mathrm{in}}^{(1)}+\pi m_{2}\end{array}$ & $\begin{array}{c}\left(1-\mathbf{e}_{r} \otimes \mathbf{e}_{r}\right) \mathbf{f}^{(2 \omega)}(\theta, \varphi)=0, \\
S_{r}^{(2 \omega)}(\theta, \varphi)=0\end{array}$ \\
\hline 11 & $\begin{array}{c}\forall \chi_{4}^{(2)}, \chi_{1-3}^{(2)}=0, \eta= \pm 1, \gamma_{1}=0, \gamma_{2}=\pi, \sigma_{1}=\sigma_{2}=0 \\
\varphi_{\mathrm{in}}^{(1)}+\varphi_{\mathrm{in}}^{(2)}=\pi m_{1}, \theta=\pi / 2, \varphi=\varphi_{\mathrm{in}}^{(1)}+\pi m_{2}\end{array}$ & $\begin{array}{l}\mathbf{f}^{(2 \omega)}(\theta, \varphi)=0 \\
S_{r}^{(2 \omega)}(\theta, \varphi)=0\end{array}$ \\
\hline 12 & $\begin{aligned} \forall \chi_{1-4}^{(2)}, \eta & = \pm 1, \gamma_{1}=0, \gamma_{2}=\pi, \sigma_{1}=\sigma_{2}, \\
\varphi_{\mathrm{in}}^{(1)}+\varphi_{\mathrm{in}}^{(2)} & =\pi m_{1}, \theta=\pi / 2, \varphi=\varphi_{\mathrm{in}}^{(1)}+\pi m_{2}\end{aligned}$ & $\begin{array}{c}\left(1-\mathbf{e}_{r} \otimes \mathbf{e}_{r}\right) \mathbf{f}^{(2 \omega)}(\theta, \varphi)=0 \\
S_{r}^{(2 \omega)}(\theta, \varphi)=0\end{array}$ \\
\hline
\end{tabular}

В соответствии с информацией в строке 5 при встречном падении электромагнитных волн $\left(\gamma_{1}=0, \gamma_{2}=\pi\right)$, имеющих одинаковые по модулю значения эллиптичности (но противоположные поляризации: $\sigma_{1}=-\sigma_{2}$ ) и совпадающие большие полуоси эллипсов поляризации $\left(\varphi_{\text {in }}^{(1)}+\varphi_{\text {in }}^{(2)}=\pi m\right)$, генерация обусловлена только первой падающей волной $\left(S_{r}^{(2 \omega)}(\theta, \varphi)=S_{r}^{(11)}(\theta, \varphi)\right)$ в направлениях, для которых выполняется условие $q^{(22)} a=Z_{1}^{(j)}$. В этом случае генерация второй гармоники и генерация суммарной частоты с участием второй волны не происходит.

Расшифровать строку 9 можно следующим образом: при встречном падении $\left(\gamma_{1}=0, \gamma_{2}=\pi\right)$ линейно поляризованных волн $\left(\sigma_{1}=\sigma_{2}=0\right)$, имеющих совпадающие плоскости поляризации $\left(\varphi_{\text {in }}^{(1)}+\varphi_{\text {in }}^{(2)}=\pi m_{1}\right)$ и произвольные амплитуды и фазы, на оптически нелинейный сферический слой, для которого характерны равные нулю коэффициенты анизотропии $\chi_{1,2}^{(2)}$, генерация не происходит в направлениях, перпендикулярных векторам электрической напряжённости падающих волн $\left(\varphi=\varphi_{\text {in }}^{(1)}+\pi / 2+\pi m_{2}\right)$.

Сгруппируем строки табл. 2 по проявлениям.

1) Генерация отсутствует при любых размерах частицы в любых направлениях: строки 1, 2.

2) Генерация отсутствует в направлении оси $O z$ : строка 3.

3) Генерация с участием одной из падающих волн отсутствует: строки 4, 5 .

4) Генерация отсутствует в отдельных направлениях, зависящих от размера частицы или только при определённых размерах частицы: строки 6, 7 .

5) Генерация отсутствует в плоскости $O x y$ : строка 8.

6) Генерация отсутствует в плоскости, перпендикулярной плоскости поляризации падающих волн: строка 9. 
Таблица 3. Комбинации параметров задачи, при которых генерируется исключительно линейно поляризованное излучение

\begin{tabular}{c|c|c}
\hline № & Комбинации параметров задачи & Вектор поляризации \\
\hline $1(2)$ & $\forall \chi_{1-3}^{(2)}, \chi_{4}^{(2)}=0, \operatorname{Im}[\eta]=0, \sigma_{1}=\sigma_{2}=0$ & $\mathbf{e}_{f}$ \\
\hline $2(3)$ & $\forall \chi_{4}^{(2)}, \chi_{1-3}^{(2)}=0, \operatorname{Im}[\eta]=0, \sigma_{1}=\sigma_{2}=0$ & $\mathbf{e}_{f}$ \\
\hline $3(6)$ & $\forall \chi_{2}^{(2)}, \chi_{1,3,4}^{(2)}=0, \operatorname{Im}[\eta]=0$, & $\mathbf{e}_{f}$ \\
\hline $4(7)$ & $\varphi_{\text {in }}^{(1)}=\pi m_{1} / 2, \varphi_{\text {in }}^{(2)}=\pi m_{1} / 2+\pi m_{2}$ & $\mathbf{e}_{f}$ \\
\hline $5(18)$ & $\gamma_{2}^{(2)}, \chi_{1,3,4}^{(2)}=0, \operatorname{Im}[\eta]=0$, & $\mathbf{e}_{\theta}$ \\
\hline $6(24)$ & $\sigma_{1}=(-1)^{m_{1}} \sigma_{2}, \varphi_{\text {in }}^{(1)}+(-1)^{m_{1}} \varphi_{\text {in }}^{(2)}=\pi m_{2}$ & $\mathbf{e}_{\varphi}$ \\
\hline $7(25)$ & $\forall \chi_{2}^{(2)}, \chi_{1,3,4}^{(2)}=0, \eta \in \mathbb{C}, \gamma_{1}=0, \gamma_{2}=\pi$ & $\mathbf{e}_{\theta}$ \\
\hline $8(26)$ & $\forall \chi_{3}^{(2)}, \chi_{1,2,4}^{(2)}=0, \eta \in \mathbb{C}, \gamma_{1}=0, \gamma_{2}=\pi, \theta=\pi / 2$ & $\mathbf{e}_{\theta}$ \\
\hline $9(27)$ & $\forall \chi_{1-4}^{(2)}, \eta \in \mathbb{C}, \gamma_{1}=0, \gamma_{2}=\pi, \sigma_{1}=\sigma_{2}=0$, & $\varphi_{\text {in }}^{(2)}+\varphi_{\text {in }}^{(2)}=\pi m_{1}, \varphi=\varphi_{\text {in }}^{(1)}+\pi / 2+\pi m_{2}$ \\
\hline $10(28)$ & $\forall \chi_{1-3}^{(2)}, \chi_{4}^{(2)}=0, \eta \in \mathbb{C}, \gamma_{1}=0, \gamma_{2}=\pi, \sigma_{1}=\sigma_{2}=0$, & $\mathbf{e}_{\varphi}^{(1)}+\varphi_{\text {in }}^{(2)}=\pi m_{1}, \varphi=\varphi_{\text {in }}^{(1)}+\pi m_{2}$ \\
\end{tabular}

7) Генерация отсутствует в направлениях, совпадающих с направлениями векторов электрической напряжённости падающих волн: строки 10-12.

Комбинации параметров задачи из табл. 2 можно использовать для оценки коэффициентов анизотропии способом, аналогичным описанному в работах $[1,3]$. Близким к нему аналогом является метод, описанный в работе [9], основанный на анализе отдельных компонент напряжённости электрического поля возбуждающего и генерируемого излучения.

Для оценки коэффициентов анизотропии можно воспользоваться, например, строкой 9. При выполнении условий из этой строки генерация отсутствует, если коэффициенты $\chi_{1}^{(2)}$ и $\chi_{2}^{(2)}$ равны нулю. Следовательно, при таких условиях генерация обусловлена только коэффициентами $\chi_{1}^{(2)}$ и $\chi_{2}^{(2)}$. Зная один из этих коэффициентов, можно оценить значение второго следующим образом. Измеряя напряжённость электрического поля генерируемого излучения при условиях из строки 9 табл. 2 и вычитая из неё напряжённость, обусловленную известным коэффициентом анизотропии и рассчитанную по формулам (15) и (20) из части I, получаем напряжённость электрического поля, обусловленную известным коэффициентом. Далее по ней можно оценить значение самого коэффициента анизотропии, пользуясь формулами (15) и (20) части I. Аналогично, зная отдельные коэффициенты анизотропии, можно определять остальные, пользуясь комбинациями параметров генерации из табл. 2.

\section{Условия линейной поляризации генерируемого излучения}

Существуют комбинации параметров генерации второй гармоники-суммарной частоты, при которых генерируемое излучение линейно поляризовано. Условия, при которых наблюдается этот эффект, указаны в табл. 3. В первом столбце табл. 3 указан номер комбинации, а в скобках номер математического свойства из табл. 1, которое выполняется при этой же комбинации параметров задачи. Во втором столбце табл. 3 указаны соответствующие комбинации параметров задачи, при которых происходит генерация линейно поляризованного излучения. В третьем столбце табл. 3 указан вектор, параллельно которому будут происходить колебания вектора электрической напряжённости генерируемого излучения. Для удобства введён вектор $\mathbf{e}_{f}$, который можно определить следующим образом:

$$
\mathbf{e}_{f}=\mathbf{e}_{\theta} \sqrt{\left(\mathbf{f}^{(2 \omega)} \mathbf{e}_{\theta}\right)\left(\mathbf{f}^{(2 \omega)} \mathbf{e}_{\theta}\right)^{*}}+\mathbf{e}_{\varphi} \sqrt{\left(\mathbf{f}^{(2 \omega)} \mathbf{e}_{\varphi}\right)\left(\mathbf{f}^{(2 \omega)} \mathbf{e}_{\varphi}\right)^{*}} .
$$

Рассмотрим некоторые строки табл. 3.

Анализируя строку 1 табл. 3, можно утверждать, что при падении двух линейно поляризованных электромагнитных волн, имеющих произвольную амплитуду и совпадающую или противоположную фазу в начале координат $(\operatorname{Im}[\eta]=0)$, на тонкий сферический нелинейный слой, не обладающий киральными свойствами $\left(\forall \chi_{1-3}^{(2)}, \chi_{4}^{(2)}=0\right)$, генерируется линейно поляризованное излучение, направление вектора электрической напряжённости которого можно определить по формуле (7). 
В этом случае линейная поляризация генерируемого излучения объясняется свойством 2 из табл. 1: вектор электрической напряжённости генерируемого излучения имеет совпадающие или противоположные фазы своих составляющих вдоль векторов $\mathbf{e}_{\theta}$ и $\mathbf{e}_{\varphi}$ $\left(\operatorname{Re}\left[\mathbf{f}^{(2 \omega)}(\theta, \varphi)\right]=0\right)$. Аналогичное свойство (строка 2 табл. 3) наблюдается и при падении электромагнитных волн на киральный слой $\left(\forall \chi_{4}^{(2)}, \chi_{1-3}^{(2)}=0\right)$. Подобные свойства были ранее замечены в отдельности для генерации второй гармоники и для генерации суммарной частоты: при падении одной или двух линейно поляризованных волн на киральный $\left(\forall \chi_{4}^{(2)}, \chi_{1-3}^{(2)}=0\right)$ или некиральный $\left(\forall \chi_{1-3}^{(2)}, \chi_{4}^{(2)}=0\right)$ слой генерируется линейно поляризованное излучение [3].

В соответствии со сведениями, приведенными в строке 5 табл. 3, при встречном падении $\left(\gamma_{1}=0, \gamma_{2}=\pi\right)$ двух электромагнитных волн, имеющих произвольную поляризацию (не записаны условия для $\sigma_{1}, \sigma_{2}$ ), амплитуду и фазу $(\eta \in \mathbb{C})$, на нелинейный сферический слой, имеющий равные нулю коэффициенты анизотропии $\chi_{1,3,4}^{(2)}$ и произвольное действительное значение коэффициента $\chi_{2}^{(2)}$, происходит генерация линейно поляризованного излучения, вектор электрической напряжённости которого направлен вдоль вектора $\mathbf{e}_{\theta}$. Это объясняется математическим свойством из строки 18 табл. 1, в соответствии с которым составляющая вектора электрической напряжённости генерируемого излучения вдоль вектора $\mathbf{e}_{\varphi}$ отсутствует $\left(\mathbf{e}_{\varphi} \mathbf{f}^{(2 \omega)}(\theta, \varphi)=0\right)$. Аналогичное свойство ранее было обнаружено в отдельности для генерации второй гармоники [1] и генерации суммарной частоты [2].

В строке 6 табл. 3 содержится информация о том, что при встречном падении $\left(\gamma_{1}=0, \gamma_{2}=\pi\right)$ двух электромагнитных волн, имеющих произвольную поляризацию, амплитуду и фазу $(\eta \in \mathbb{C})$, на тонкий оптически нелинейный слой, имеющий равные нулю коэффициенты анизотропии $\chi_{1,2,4}^{(2)}$ и произвольное действительное значение коэффициента $\chi_{3}^{(2)}$, происходит генерация линейно поляризованного излучения в плоскости $O x y(\theta=\pi / 2)$. При этом вектор электрической напряжённости генерируемого излучения направлен вдоль вектора $\mathbf{e}_{\varphi}$.

Свойства, указанные в табл. 3, можно также наблюдать на диаграммах направленности, проиллюстрированных в части II статьи:

- свойство 1 проявляется на рис. 2, $a-c, e$, на рис. $3, a, b, d, f$ и на рис. $8, a, b, d, f$;

- свойство 2 проявляется на рис. $2, d$.

Сгруппируем строки табл. 3 по проявлению свойств.

1) Составляющие вектора электрического поля генерируемого излучения вдоль векторов $\mathbf{e}_{\theta}$ и $\mathbf{e}_{\varphi}$ имеют одинаковые фазы: строки 1-4.

2) Отсутствует составляющая вектора электрического поля генерируемого излучения вдоль вектора $\mathbf{e}_{\varphi}$ : строка 5.
3) В плоскости $O x z$ отсутствует составляющая вектора электрической напряжённости генерируемого излучения вдоль вектора $\mathbf{e}_{\theta}$ или $\mathbf{e}_{\varphi}$ : строки 6 или 10 соответственно.

4) В плоскости, перпендикулярной плоскости поляризации падающих волн, отсутствует составляющая вектора электрической напряжённости генерируемого излучения вдоль вектора $\mathbf{e}_{\varphi}$ : строка 7.

5) В плоскости, совпадающей с плоскостью поляризации падающих волн, отсутствует составляющая вектора электрической напряжённости генерируемого излучения вдоль вектора $\mathbf{e}_{\varphi}$ или $\mathbf{e}_{\theta}$ : строки 8 или 9 соответственно.

Описанные условия генерации линейно поляризованного излучения также можно использовать для оценки коэффициентов анизотропии. Например, в соответствии со свойством в строке 8 , если киральный коэффициент $\chi_{4}^{(2)}=0$ и выполняются условия из второго столбца этой строки, то составляющая вектора электрической напряжённости генерируемой волны, направленная вдоль вектора е описанные условия нарушены). Следовательно, модуль этой составляющей зависит только от коэффициента $\chi_{4}^{(2)}$ и не зависит от остальных $\chi_{1-3}^{(2)}$. Измеряя эту составляющую и пользуясь формулами (15) и (20) из части I, можно оценить значение этого коэффициента.

На основе информации в строке 10 получаем, что, зная коэффициент анизотропии $\chi_{1}^{(2)}$, можно оценить значение коэффициента $\chi_{3}^{(2)}$. Модуль составляющей вектора электрической напряжённости генерируемого поля вдоль вектора $\mathbf{e}_{\varphi}$ зависит от коэффициентов $\chi_{1}^{(2)}$ и $\chi_{3}^{(2)}$ при выполнении условий из строки 10. Если измерить величину электрической напряжённости генерируемого излучения вдоль вектора $\mathbf{e}_{\varphi}$ и вычесть вклад коэффициента $\chi_{3}^{(2)}$, рассчитанный по формулам (15) и (20) из части I, то полученное значение будет зависеть только от коэффициента $\chi_{1}^{(2)}$; и наоборот: зная коэффициент $\chi_{1}^{(2)}$, с помощью аналогичных действий можно оценить коэффициент $\chi_{3}^{(2)}$, вычитая вклад, обусловленный коэффициентом $\chi_{1}^{(2)}$. Подобным образом, зная отдельные коэффициенты анизотропии, можно определять остальные с использованием свойств в табл. 3.

\section{Свойства, связанные с заменой параметров}

Рассмотрим свойства симметрии, которые проявляются при замене отдельных параметров задачи. Первые два свойства можно наблюдать только в случаях, когда нелинейный слой является некиральным $\left(\chi_{1-3}^{(2)} \neq 0, \chi_{4}^{(2)}=0\right)$ или киральным $\left(\chi_{1-3}^{(2)}=0, \chi_{4}^{(2)} \neq 0\right)$. Новые значения параметров обозначены символом $\sim$ сверху.

Свойство 1. При подстановке новых значений параметров задачи

$$
\tilde{\eta}=\eta^{*}, \quad \tilde{\sigma}_{1}=-\sigma_{1}, \quad \tilde{\sigma}_{2}=-\sigma_{2},
$$


выраженных через первоначальные, векторы и функции в задаче меняются следующим образом:

$$
\tilde{\mathbf{e}}^{(\alpha)}=\left[\mathbf{e}^{(\alpha)}\right]^{*}, \quad \tilde{S}_{r}^{(2 \omega)}(\theta, \varphi)=S_{r}^{(2 \omega)}(\theta, \varphi) .
$$

При этом для некирального слоя $\left(\chi_{1-3}^{(2)} \neq 0, \chi_{4}^{(2)}=0\right)$

$$
\tilde{\mathbf{f}}^{(2 \omega)}(\theta, \varphi)=-\left[\mathbf{f}^{(2 \omega)}(\theta, \varphi)\right]^{*},
$$

а для кирального $\left(\chi_{1-3}^{(2)}=0, \chi_{4}^{(2)} \neq 0\right)$

$$
\tilde{\mathbf{f}}^{(2 \omega)}(\theta, \varphi)=\left[\mathbf{f}^{(2 \omega)}(\theta, \varphi)\right]^{*} .
$$

Свойство 2. Переходя к новым значениям параметров

$$
\tilde{\sigma}_{1}=-\sigma_{1}, \quad \tilde{\sigma}_{2}=-\sigma_{2}, \quad \tilde{\varphi}_{\text {in }}^{(1)}=-\varphi_{\text {in }}^{(1)}, \quad \tilde{\varphi}_{\text {in }}^{(2)}=-\varphi_{\text {in }}^{(2)},
$$

можем обнаружить, что выполняется следующее равенство:

$$
\tilde{S}_{r}^{(2 \omega)}(\theta, \varphi)=S_{r}^{(2 \omega)}(\theta, \pi-\varphi) .
$$

Для некирального слоя $\left(\chi_{1-3}^{(2)} \neq 0, \chi_{4}^{(2)}=0\right)$ в этом случае

$$
\tilde{\mathbf{f}}^{(2 \omega)}(\theta, \varphi)=\left(1-2 \mathbf{e}_{\varphi} \otimes \mathbf{e}_{\varphi}\right) \mathbf{f}^{(2 \omega)}(\theta, \pi-\varphi),
$$

а для кирального $\left(\chi_{1-3}^{(2)}=0, \chi_{4}^{(2)} \neq 0\right)$

$$
\tilde{\mathbf{f}}^{(2 \omega)}(\theta, \varphi)=-\left(1-2 \mathbf{e}_{\varphi} \otimes \mathbf{e}_{\varphi}\right) \mathbf{f}^{(2 \omega)}(\theta, \pi-\varphi) .
$$

Указанное свойство, как и предыдущее, связано с возможностью замены левоэллиптически поляризованных исходных волн на правоэллиптически поляризованные.

Свойство 3. После подстановки следующих значений параметров задачи:

$$
\tilde{\eta}=\frac{1}{\eta}, \quad \tilde{\sigma}_{1}=\sigma_{2}, \quad \tilde{\sigma}_{2}=\sigma_{1}, \quad \tilde{\varphi}_{\text {in }}^{(1)}=\varphi_{\text {in }}^{(2)}, \quad \tilde{\varphi}_{\text {in }}^{(2)}=\varphi_{\text {in }}^{(1)}
$$

новые значения векторов и функций также можно выразить через старые:

$$
\begin{gathered}
\tilde{\mathbf{q}}^{(11)}(\theta, \varphi)=\mathbf{q}^{(22)}(\theta, \varphi+\pi), \quad \tilde{\mathbf{q}}^{(12)}(\theta)=\mathbf{q}^{(12)}(\theta), \\
\tilde{\mathbf{q}}^{(22)}(\theta, \varphi)=\mathbf{q}^{(11)}(\theta, \varphi+\pi), \\
\tilde{\mathbf{e}}^{(1)}(\theta, \varphi)=-\mathbf{e}^{(2)}(\theta, \varphi+\pi), \\
\tilde{\mathbf{e}}^{(2)}(\theta, \varphi)=-\mathbf{e}^{(1)}(\theta, \varphi+\pi), \\
\tilde{\mathbf{f}}^{(11)}(\theta, \varphi)=\mathbf{f}^{(22)}(\theta, \varphi+\pi), \quad \tilde{\mathbf{f}}^{(12)}(\theta, \varphi)=\mathbf{f}^{(12)}(\theta, \varphi+\pi), \\
\tilde{\mathbf{f}}^{(22)}(\theta, \varphi)=\mathbf{f}^{(11)}(\theta, \varphi+\pi), \\
\tilde{\mathbf{f}}^{(2 \omega)}(\theta, \varphi)=\eta^{-2} \mathbf{f}^{(2 \omega)}(\theta, \varphi+\pi), \\
\tilde{S}_{\text {norm }}^{(2 \omega)}(\theta, \varphi)=S_{\text {norm }}^{(2 \omega)}(\theta, \varphi+\pi),
\end{gathered}
$$

где

$$
S_{\text {norm }}^{(2 \omega)}(\theta, \varphi)=\frac{S_{r}^{(2 \omega)}(\theta, \varphi)}{\left(1+|\eta|^{2}\right)^{2}}
$$

- нормированное значение плотности мощности генерируемого излучения, которое будет описано более подробно в части IV данной статьи. Свойства (17)-(19) обусловлены возможностью взаимной перестановки местами первой и второй исходных волн.

Свойство 4. Если $\gamma=0$, то, подставляя новые значения параметров

$$
\tilde{\eta}=\frac{1}{\eta^{*}}, \quad \tilde{\sigma}_{1}=\sigma_{2}, \quad \tilde{\sigma_{2}}=\sigma_{1}
$$

в результирующие формулы, получим

$$
\begin{aligned}
\tilde{\mathbf{f}}^{(2 \omega)}(\theta, \varphi)= & -\left(1-2 \mathbf{e}_{\varphi} \otimes \mathbf{e}_{\varphi}\right) \\
& \times\left(\eta^{-2} \mathbf{f}^{(2 \omega)}\left(\theta, \varphi_{\text {in }}^{(1)}+\varphi_{\text {in }}^{(2)}+\pi-\varphi\right)\right)^{*}, \\
\tilde{S}_{\text {norm }}^{(2 \omega)}(\theta, \varphi)= & S_{\text {norm }}^{(2 \omega)}\left(\theta, \varphi_{\text {in }}^{(1)}+\varphi_{\text {in }}^{(2)}+\pi-\varphi\right) .
\end{aligned}
$$

Данное свойство связано с возможностью перестановки первой и второй волн местами без изменения ориентаций эллипсов поляризации для сонаправленных волн и объясняется поворотом эквивалентного вектора поляризации при переходе к генерации второй гармоники (формула (30) части I).

Свойство 5. При $\gamma=\pi, \sigma_{1}= \pm 1, \sigma_{2}= \pm 1$ следствием выполнения замены

$$
\tilde{\eta}=\frac{1}{\eta^{*}}
$$

является равенство

$$
\tilde{S}_{\text {norm }}^{(2 \omega)}(\theta, \varphi)=S_{\text {norm }}^{(2 \omega)}(\pi-\theta, \varphi) .
$$

Отметим, что последние три свойства выполняются при любых значениях тензора нелинейной диэлектрической восприимчивости.

\section{Заключение}

Установленные математические свойства дополняют решение задачи о генерации второй гармоники-суммарной частоты в тонком слое, расположенном на поверхности сферической диэлектрической частицы. В них отражено влияние параметров генерации на характеристики генерируемого излучения, такие как симметрии и особенности пространственного распределения поля, в том числе отдельных его компонент. Обнаруженные свойства также отражают связь рассмотренного в работе явления с гораздо более часто обсуждаемым эффектами: генерацией второй гармоники и генерацией суммарной частоты. Симметрия задачи также проявляется в возможности перестановки исходных волн местами и смены поляризации возбуждающего излучения на противоположную без существенного изменения формы диаграммы направленности генерируемого излучения.

Условия отсутствия генерации и условия генерации линейно поляризованного излучения могут найти применение при определении компонент тензора нелинейной диэлектрической восприимчивости поверхностного слоя, связанных в свою очередь со свойствами адсорбированных на поверхности частицы молекул, например пространственной ориентацией и плотностью 
распределения. Симметрии, выявленные в работе, могут применяться при планировании эксперимента, когда в силу симметричности ожидаемой картины распределения излучения пропадает необходимость производить измерения в некоторых направлениях.

\section{Финансирование работы}

Работа выполнена при финансовой поддержке Белорусского республиканского фонда фундаментальных исследований (грант Ф20М-011).

\section{Конфликт интересов}

Авторы заявляют, что у них нет конфликта интересов.

\section{Список литературы}

[1] Капшай В.Н., Шамына А.А. // Опт. и спектр. 2017. T. 123. № 3. C. 416. doi $10.7868 / \mathrm{S} 003040341709015 \mathrm{X}$; Kapshai V.N., Shamyna A.A. // Opt. Spectrosc. 2017. V. 123. N 3. P. 440. doi 10.1134/S0030400X17090144

[2] Шамына А.А., Капшай В.Н. // Опт. и спектр. 2018. T. 125. № 1. C. 71. doi 10.21883/OS.2018.07.46269.56-17; Shamyna A.A., Kapshai V.N. // Opt. Spectrosc. 2018. V. 125. N 1. P. 74. doi 10.1134/S0030400X1807024X

[3] Капшай В.Н., Шамына А.А. // Опт. и спектр. 2019. T. 126. № 6. C. 732. doi 10.21883/OS.2019.06.47766.375-18; Kapshai V.N., Shamyna A.A. // Opt. Spectrosc. 2019. V. 126. N 6. P. 653. doi 10.1134/S0030400X19060134

[4] Шамына А.А., Капшай В.Н. // Опт. и спектр. 2019. T. 126. № 6. C. 724. doi 10.21883/OS.2019.06.47767.376-18; Shamyna A.A., Kapshai V.N. // Opt. Spectrosc. 2019. V. 126. N 6. P. 661. doi 10.1134/S0030400X19060237

[5] Шамына А.А., Капшай В.Н. // Опт. и спектр. 2018. T. 124. № 1. C. 105. doi 10.21883/OS.2018.01.45366.176-17; Shamyna A.A., Kapshai V.N. // Opt. Spectrosc. 2018. V. 124. N 1. P. 103. doi $10.1134 / \mathrm{S} 0030400 X 18010198$

[6] Tang Y., Cohen A.E. // Science. 2011. V. 332. N 6027. P. 333. doi 10.1126/science. 1202817

[7] Kriech M.A., Conboy J.C. // J. Opt. Soc. Am. B. 2004. V. 21. N 7. P. 1013. doi 10.1364/JOSAB.21.001013

[8] Dadap J.I., Shan J., Heinz T.F. // J. Opt. Soc. Am. B. 2004. V. 21 . N 7. P. 1328 . doi $10.1364 / J O S A B .21 .001328$

[9] De Beer A.G.F., Roke S. // Phys. Rev. B. 2007. V. 75. N 24. P. 245438. doi 10.1103/PhysRevB.75.245438 